\title{
Nitric Oxide Synthases of Bacteria - and Other Unicellular Organisms
}

\author{
Steven Rafferty* \\ Department of Chemistry, Trent University, 1600 West Bank Drive, Peterborough, ON Canada K9J 7B8
}

\begin{abstract}
The defining feature of a nitric oxide synthase (NOS) is a heme and pterin-binding oxygenase domain, and enzymes that possess this domain are found in animals and bacteria. While the roles of animal NOS in such processes as vasodilation, neurotransmission, and host defence are understandably important for multicellular life, they are meaningless in bacteria, which makes bacterial NOSs all the more interesting as objects of study. This review describes the structural similarities and differences between animal and bacterial NOS, and how such differences may influence the extent and duration of bacterial NO production, and, ultimately the roles of NO in microorganisms. Recent progress in defining the functions of bacterially-derived $\mathrm{NO}$, notably in protection from various stresses and as a potential transcriptional regulator are described. Finally, an attempt is made to span the gap between animal and bacterial nitric oxide synthases by commenting on the search for examples of NOS in single-celled eukaryotes, where progress seems imminent.
\end{abstract}

Keywords: Bacterial nitric oxide synthase, unicellular nitric oxide synthase, nitric oxide synthase structure and function.

\section{INTRODUCTION}

The story of nitric oxide synthase (NOS) is a wonderful example of how an enzyme's function can evolve over time, even as its structure and mechanism remain largely intact. Constitutive NOS isotypes of mammals are used for the transient production of nanomolar amounts of NO for neurotransmission and vasodilation, while at the higher concentrations produced by inducible NOS in macrophages over sustained periods, NO is cytotoxic and is used to kill pathogens. Yet the origin of NOS is ancient; since the turn of the century the number of bacteria known to carry a NOS gene has increased sharply, and the work of defining the roles of bacterial NOS is an active and exciting area of research. But the story of NOS is also the story of an enzyme that has been characterized mainly in these two distinct kingdoms, the metazoans (overwhelmingly of mammals) and the bacteria, with few studies from other branches of life. If the bacteria originated it and we still have it, why is it seemingly so rare elsewhere? If it can be found in other kingdoms, for example among the protists, what might its functions be?

This brief review will begin with the features common to NOS and then, gradually, move towards their differences. This includes the biochemical targets of NO, the reaction catalyzed by NOS, common structural features and differences in the enzyme, and the different roles that these enzymes have, with the focus on bacterial NOSs. Having considered these aspects, the prospects of the presence of NOS in single-celled eukaryotes and their possible functions will be discussed, which, because of the paucity of

*Address correspondence to this author at the Department of Chemistry, Trent University, 1600 West Bank Drive, Peterborough, ON Canada K9J 7B8; Tel: 705-748-1011; Ext: 7309; E-mail: srafferty@trentu.ca information, will be brief and speculative rather than deep and factual.

\section{BIOCHEMICAL TARGETS OF NO}

As a freely-diffusible and neutral gas, NO is highly mobile and can act within the cell that produces it as well as in adjacent cells. NO is capable of reacting directly with certain biomolecules and indirectly, through reactive nitrogen species that are derived from it. NO is a potential ligand to iron-containing complexes such as heme and ironsulfur centres; it acts as an axial ligand to ferriheme and forms particularly strong coordinate bonds with ferroheme proteins. This interaction is important in the mammalian signalling roles of $\mathrm{NO}$, in which NO binds to a ferrohemecontaining subunit on soluble guanylate cyclase and activates it $[1,2]$. NO can also directly nitrosylate nonheme iron centres in iron-sulfur clusters [3]. While this can be detrimental to respiratory proteins and enzymes which have such centres, it is also an important way of controlling gene expression, and several bacterial transcription factors also possess iron-sulfur clusters that render their activity sensitive to NO $[4,5]$. NO can also react with and quench the active site tyrosyl radical of ribonucleotide reductase, which produces deoxyribonucleotides from ribonucleotides [6].

Apart from these examples of the direct reaction of $\mathrm{NO}$ with biological targets are the actions of reactive nitrogen species derived from NO. These include the nitrosonium ion $\mathrm{NO}^{+}$, which can react with biological thiols such as glutathione and cysteine residues to form S-nitroso compounds [7]. These compounds are long-lived and stable, and can extend the effects of NO far beyond the site of its generation. $\mathrm{NO}$ also reacts with molecular oxygen to form nitrite and nitrate, and with superoxide to form highly reactive and damaging peroxynitrite [8]. 
With many possible reactions, the biological effects of NO are rarely simple, and will depend not only on the rate of its production or the manner in which it is delivered (endogenously catalyzed by NOS, or exogenously delivered as NO gas or by an NO-donor compound), but on the presence of other nearby species such as oxygen, radicals, redox-active metal centres and thiols. An additional consideration is the site of NO generation. Endothelial and neuronal NOSs are targeted to specific locations within the cell, and errors in targeting can have biological consequences, even if the enzyme activity is not altered [9$11]$.

\section{COMMON FEATURES OF NITRIC OXIDE SYNTHASES}

A nitric oxide synthase is defined by its structure, function, and mechanism. Structurally a NOS is a homodimeric heme protein with axial thiolate coordination, and a pterin cofactor (either tetrahydrobiopterin or tetrahydrofolate), bound between the propionyl groups of the heme; the protein fold is unique to this enzyme class [12, 13]. NOSs catalyze the oxidation of L-arginine to citrulline and NO using molecular oxygen and reducing equivalents provided by nicotinamide cofactors through the mediation of a reductase domain, which may be a separate enzyme or a module on the same protein chain. As the multidomain enzymes from mammals were the first to be discovered and studied, the term NOS was used to describe the entire enzyme, while "NOS oxygenase domain", or NOS oxy $_{\text {}}$ denoted the heme-and pterin binding module. All known bacterial NOSs, with one notable exception described below, consist solely of the oxygenase domain $[14,15]$.

The NOS-catalyzed reaction occurs in two steps through the intermediate N-hydroxy-L-arginine (NOHA) [16]. The first step is a monoxygenation in which one atom of molecular oxygen is inserted into L-arginine and the other is reduced to water using electrons and protons provided by a reduced nicotinamide cofactor through a reductase. Thiolate coordination to a heme cofactor, and the ability to catalyze monooxygenation reactions are also features of the cytochromes P450, and the mechanism of NOS has some similarities to this enzyme class, although in other structural respects the two are distinct. The mechanism of NOS has been reviewed recently, and our understanding remains incomplete, especially for the set of reactions leading from NOHA to NO and citrulline $[17,18]$. The first half of the NOS-catalyzed reaction to make NOHA likely proceeds through a Compound I-type intermediate with heme and a bound oxygen atom in an oxyferryl porphyrin $\pi$-cation radical, as is thought to occur in the cytochrome P450s. There is more debate regarding the nature of the second step; although a Compound I state has been suggested, there is also evidence that the active species is a ferric peroxy intermediate that acts as a nucleophile on NOHA to yield citrulline and NO. The participation of the pterin cofactor as an electron donor and acceptor in the reaction cycle demonstrates that the NOS mechanism can not be treated merely as that of conventional cytochrome P450, as these do not depend on pterins for their activity.

In mammalian NOS the pterin used is exclusively tetrahydrobiopterin $\left(\mathrm{H}_{4} \mathrm{~B}\right)$, while in bacteria tetrahydrofolate
$\left(\mathrm{H}_{4} \mathrm{~F}\right)$ may also be used. In either case the pteridine ring structure is the same, and both serve the same role in donating an electron to the ferrous-oxy intermediate, which leaves the pteridine ring in an unusual radical cation state $\left(\mathrm{H}_{4} \mathrm{~B}^{+} \cdot\right)[19,20]$. This role is carried out in both the formation of NOHA and its subsequent reaction, but the fate of the radical differs. While in the first step the radical is reduced by electrons supplied by the reductase partner, ferrous heme reduces the radical in the second step. This is critical to the function of NOS, as ferroheme has a high affinity for NO, which would otherwise autoinhibit the enzyme after one reaction cycle [19]. Electron transfer from the ferroheme-NO complex to $\mathrm{H}_{4} \mathrm{~B}^{+}$. generates the labile ferriheme-NO complex.

\section{STRUCTURAL DIFFERENCES BETWEEN NITRIC OXIDE SYNTHASES}

These structural and mechanistic features described above are common to all NOSs that have been studied to date, which include the three mammalian isotypes (neuronal, endothelial, inducible) as well as several from bacteria, notably from Bacillus subtilis, Bacillus anthracis, Staphylococcus aureus, Deinococcus radiodurans, and Sorangium cellulosum. Key residues in the cofactor and substrate binding sites are well-conserved among these enzymes, as well as in the coding sequences of NOS from the genomes of other organisms. Still, there are notable structural differences within the NOS oxygenase domain and in the nature and location of the supporting reductase domain as noted below, in Table 1, and in Fig. (1).

Although similar in sequence to their mammalian counterparts and sharing up to $47 \%$ sequence identity, most bacterial NOSs lack a 100-residue amino-terminal segment that forms part of the $\mathrm{H}_{4} \mathrm{~B}$-binding site and dimer interface of mammalian $\mathrm{NOS}_{\text {oxy }}$, and which also includes an interfacial, tetrathiolate-coordinated $\mathrm{Zn}(\mathrm{II})$. While this segment is essential for dimer formation in mammalian NOSs, the bacterial enzymes form stable dimers without it, owing to compensatory changes elsewhere in the dimer interface [14]. The absence of this domain renders the pterin binding site of the bacterial NOSs more solvent accessible, and accommodating to other molecules. The $\mathrm{H}_{4} \mathrm{~B}$ cofactor found in the mammalian enzymes can bind tightly, but so can $\mathrm{H}_{4} \mathrm{~F}$ and $\mathrm{NAD}^{+}[14,15]$, and possibly tryptophan. This ability to accommodate other molecules at the pteridine binding site endows the bacterial NOSs with additional catalytic abilities beyond $\mathrm{NO}$ synthesis.

A second difference between bacterial and animal NOSs is sequence variation within the common oxygenase domain structure. While many active site residues around the heme cofactor are conserved between bacterial and animal NOS, some residues are associated with one class but not the other. These structural differences can lead to differences the rates of individual catalytic steps between bacterial and mammalian enzymes [21-23]. For example, valine-346 (mouse iNOS numbering) is conserved in mammalian NOSs, and is located above the distal face of the heme; in bacteria an isoleucine residue occupies this position (isoleucine- 218 in bsNOS). This residue influences the rate of ligand dissociation from the heme, with the smaller valine residue linked to higher rates. The role of this residue in controlling 
Table 1. Major Differences Between Mammalian and Bacterial NOSs

\begin{tabular}{|c|c|c|}
\hline $\begin{array}{l}\text { Oxygenase/reductase domain } \\
\text { organization }\end{array}$ & $\mathrm{N}$-terminal oxygenase, $\mathrm{C}$-terminal reductase domains & $\begin{array}{l}\text { Oxygenase domain only, } \\
\text { multiple reductase partners* }\end{array}$ \\
\hline Oxygenase domain dimer & Stabilized by $\mathrm{Zn}^{2+}$ bound at the dimer interface & Lacks $\mathrm{Zn}^{2+}$ binding site but forms stable dimers \\
\hline Pterin binding site & Closed; can only fit $\mathrm{H}_{4} \mathrm{~B}$ & Exposed; can hold $\mathrm{H}_{4} \mathrm{~B}, \mathrm{H}_{4} \mathrm{~F}$, and other molecules \\
\hline Regulation & $\begin{array}{l}\mathrm{Ca}^{2+} / \mathrm{CaM} \text { (NOS1, NOS3) } \\
\text { Transcriptionally (NOS2) }\end{array}$ & Unknown \\
\hline Roles of NO & $\begin{array}{l}\text { Neurotransmission (NOS1), Vasodilation (NOS3), } \\
\text { Host defence (NOS2) } \\
\text { and others }\end{array}$ & $\begin{array}{l}\text { Counters oxidative stress, } \\
\text { Counters UV damage, } \\
\text { Biosynthesis of nitrated compounds, } \\
\text { Transcriptional regulation }\end{array}$ \\
\hline
\end{tabular}

*With the exception of scNOS, as noted in the text.

the rate of exogenous ligand dissociation was confirmed by the properties of the mouse iNOS V346I and bsNOS I218V mutants [24]. The rate of NO release from the mammalian NOS was decreased nearly three-fold, while that of the bacterial mutant increased four-fold, over their respective wild types. This modest structural difference contributes to the generally lower catalytic activity of bacterial NOSs compared to their animal counterparts, a difference that is likely related to the different roles of bacterially-generated NO. Further differences in the properties of bacterial and mammalian NOS oxygenase domains are revealed by techniques such as resonance-Raman spectroscopy, which has been an especially sensitive probe of the heme environment under a variety of conditions [25-27].

A third structural difference is the nature of the reductase domain and whether this is linked to the oxygenase domain (Fig. 1). Mammalian NOSs are large, multimodule enzymes with an amino-terminal oxygenase domain linked to an FMN and FAD-containing reductase domain, which binds NADPH and transfers electrons through the flavin cofactors to the oxygenase domain [28]. Structurally and functionally, this fused reductase domain resembles a cytochrome P450 reductase. An intervening calmodulin-binding segment lies between the oxygenase and reductase domains; this segment, along with the presence of an autoinhibitory loop within the FMN-binding portion of the reductase domains of the mammalian endothelial and neuronal isotypes, are key components in controlling their activity through $\mathrm{Ca}(\mathrm{II}) /$ calmodulin interactions.

Additional differences are found in the amino-terminal domains of the mammalian NOS isotypes, which in the neuronal and endothelial isotypes are involved in targeting the proteins to their proper subcellular locations. Neuronal NOSs possess a PDZ domain which interacts with the postsynaptic density-95 protein found at synaptic junctions in neuronal cells [10], while endothelial NOSs have two sites for acylation and one for phosphorylation, which are important in the localization of this isotype to the caveolae, a type of lipid raft on the plasma membrane of vascular endothelial cells $[29,30]$.
This particular domain organization of the mammalian NOSs is shared by all metazoan enzymes, as inferred by their DNA sequences. The evolution of metazoan NOS isotypes is not straightforward, but the essential multidomain organization of an amino-terminal oxygenase domain linked through a calmodulin binding site to an FMN and FADbinding reductase domain seems to have been established more than 800 million years ago [31].

The complexity of the metazoan NOSs is in contrast to the simplicity of most bacterial counterparts, which consist solely of a homodimeric oxygenase domain. Although these require a separate supporting reductase enzyme, most single domain bacterial NOSs are not particular in their choice of partner, and they can accept electrons from several types of endogenous $\mathrm{NAD}(\mathrm{P}) \mathrm{H}$ oxidoreductases found within the bacterial cell, or from recombinant mammalian NOS reductase domains in vitro [32-34].

To state that all metazoan NOSs are multidomain enzymes while bacterial versions are single-domain is not accurate. Splice variants of mammalian NOS which produce truncated versions consisting only of the oxygenase domain have been detected, and these can form heterodimers with the full-length protein and act in a dominant-negative fashion to suppress NOS activity [35, 36]. Conversely, bacterial NOSs can also be multidomain enzymes, as was demonstrated by recent characterization of NOS from Sorangium cellulosum [37]. This enzyme is noteworthy for being the first multidomain bacterial NOS to be discovered, and the first isolated from a Gram-negative bacteria. ScNOS has a reductase domain that accepts electron from NAD $(\mathrm{P}) \mathrm{H}$ through an FNR-like FAD-binding module and a ferredoxin module which bears an $\mathrm{Fe}_{2} \mathrm{~S}_{2}$ cluster. This reductase domain also differs from the mammalian enzymes in that it occurs at the amino-terminal end of the protein chain, rather than the carboxy-terminal end as found in metazoan NOS. Whether scNOS is unique among the bacterial NOSs in its modular structure remains to be seen.

\section{CONTROL OF NITRIC OXIDE LEVELS}

The purpose of NO is related to the context of its production and consumption: the quantity of NO made, the 
Fig. (1). Domain organization of nitric oxide synthases. NOSs are defined by the presence of a core oxygenase domain (blue) that binds heme and pterin and is the catalytic centre of the enzyme. In most bacteria (top) this is the complete protein, and it receives electrons from separate reductase domains. The sole known bacterial exception is Sorangium cellulosum NOS (scNOS, middle), which has an aminoterminal reductase domain (red). In the metazoa (bottom) the domain structure is more elaborate; from the amino to carboxy termini this comprises an isotype-specific targeting domain (yellow), a $\mathrm{Zn}^{2+}$-binding subdomain (cyan) that stabilizes the oxygenase domain (blue), a calmodulin binding domain (CBD, grey) and a reductase domain (red). The reductase domain of scNOS is homologous to a ferredoxin nucleotide reductase /ferredoxin fusion, while that of the metazoan NOS is homologous to cytochrome P450 reductase. Both scNOS and metazoan NOS receive electrons from NADPH. All NOSs form dimers through interactions between the oxygenase domains.

duration of production, the nature of nearby targets that can react with and break down NO are all important. These will depend in part on the nature of the enzyme itself, specifically its location within the cell and its domain organization; it will also depend on the control of the expression level of the protein and the availability of cofactors.

The structural distinction between multidomain metazoan NOSs, and the single-domain Gram positive bacterial enzymes which accept electrons from a variety of reductase partners has important functional consequences. Selfcontained, multidomain NOSs have the potential for rapid and reversible control of activity by gating electron transfer between domains. The best examples of this are the mammalian constitutive NOS isotypes that are controlled by calcium ion concentration. All mammalian NOSs have a calmodulin binding site between the N-terminal oxygenase and $\mathrm{C}$-terminal reductase domains, and the constitutive endothelial and neuronal isotypes have a well understood and efficient mechanism for controlling enzyme activity by $\mathrm{Ca}(\mathrm{II}) /$ calmodulin binding, which increases the rate of electron transfer between reductase and oxygenase domains. Furthermore, an autoinhibitory loop found within the FMNbinding module of the reductase domain, also prevents interdomain electron transfer unless $\mathrm{Ca}$ (II)-loaded calmodulin is bound to the enzyme. In these cases, NO production can be rapidly and reversibly controlled through transient fluctuations in $\mathrm{Ca}$ (II) concentration (ranging from the nanomolar to micromolar level), and toxic levels of NO can be avoided. While the most thoroughly studied examples are the mammalian enzymes, similar enzymes are also encoded by most other metazoans where they presumably have the same characteristic of rapid and reversible control of NO production. Even the inducible NOS2 isotype, which has an activity independent of $\mathrm{Ca}$ (II) concentration, retains a tight calmodulin binding site, although not the autoinhibitory loop. Presumably the ancestral sequence of the NOS2 isotype was a calcium-sensitive enzyme which lost its ability to respond to $\mathrm{Ca}(\mathrm{II})$.

The mammalian isotypes define the two extremes of NO production. On the one hand, endothelial and neuronal NOS isotypes have tight, rapid, and reversible control of small amounts of NO in targeted locations within the cell. This is ideal for signalling roles that require a rapid response, similar to the action of a light switch in a room. In contrast, inducible NOS, while sharing a similar domain structure, is like a floodlight, that once plugged in cannot be easily turned off. The NO produced by these isotypes is used for starkly different purposes: rapid signalling on the one hand, cytotoxicity on the other.

Less is known about the control of NO production in the bacteria that possess a single-domain NOS, but it likely lies between the extremes of the mammalian NOSs, producing more NO over longer periods than endothelial and neuronal NOS, but less than that of the inducible isotype. Rapid and precise control may not be possible with these forms of bacterial NOS, as it is unlikely that intermolecular electron transfer could occur as quickly as intramolecular electron transfer. By having the oxygenase and reductase domains as separate proteins, the amount of NO produced in a cell might be lower, and may be limited by the rates at which these proteins encounter one another. Furthermore, precise regulation of NO production would seem to require a NOSspecific reductase partner that would only transfer electrons when required to do so, but bacterial NOSs can accept electrons from a variety of endogenous partners in vivo.

As several bacterial reductases can interact with NOS, and these presumably have other alternate electron transfer partners, the control of bacterial NOS activity, if it occurs at all, would likely be exerted by changes on the NOS oxygenase domain alone. Control could conceivably be exerted through modification of the bacterial NOS, either through covalent modification of the enzyme, control of $\mathrm{H}_{4} \mathrm{~F}$ availability, or a conformational change between active and inactive states. All of these are possible but none have been established with certainty. Bacteria possess kinase and phosphatase systems that modify specific hydroxylcontaining residues on their target proteins [38, 39], but whether bacterial NOSs are so modified is not known. Pterins are essential cofactors for NOS activity, and it is interesting that the binding affinities for $\mathrm{H}_{4} \mathrm{~F}$ and $\mathrm{H}_{4} \mathrm{~B}$ of the 
bacterial NOSs are generally one to two orders of magnitude lower than for $\mathrm{H}_{4} \mathrm{~B}$ binding to the mammalian NOS, and are comparable to those of the substrate, L-arginine [22]. $\mathrm{As}_{4} \mathrm{~F}$ is required for NOS activity, cofactor (and L-arginine) availability may be a means of enzyme control in the bacterial isotypes. This may also be linked to control via conformational changes within the enzyme; the Bacillus subtilis NOS dimer exists in two different conformations, a less active 'loose' conformation and a more active 'tight' state, with the latter favored in the presence of L-arginine or $\mathrm{H}_{4} \mathrm{~B}[40]$.

As a self-contained enzyme, the activity of Sorangium cellulosum NOS has the potential to be regulated through the control of interdomain electron transfer, but the way this is accomplished (if such control exists) is unknown. A sequence of $\sim 50$ amino acid residues links the reductase domain to the oxygenase domain. A BLAST search of this sequence (amino acids 750-800) does not identify any related sequences, and the sequence itself (DAIQRELFSSNVDTAGEVRPTPLRRAGAARAARAAG GVCPVEHGSFHVTPT) is predicted to be a central, basic alpha helix (underlined) flanked by random coil [41]. This is similar in length and in charge properties to the calmodulinbinding linker between the oxygenase and reductase domains of mammalian NOSs. This similarity may be coincidental, as bacteria lack calmodulin, and the central helix is not a canonical calmodulin binding site. Yet one may still speculate on its possible regulatory role. Recombinant $S$. cellulosum NOS is active in the absence of any other protein, which suggests that the role of the linking sequence above, if it has any regulatory role at all, would be to bind a protein that would inhibit interdomain electron transfer.

Another potential means of regulation of scNOS is through control of quaternary structure. Gel filtration chromatography of recombinant scNOS demonstrates that it is a mixture of monomer and dimer, while recombinant scNOSoxy is exclusively dimeric, indicating that interactions of the reductase domain with the oxygenase domain can destabilize the active dimer structure under certain circumstances. In this study, it does not appear that Larginine or pterin cofactor were included in the gel filtration chromatography buffers. It would be interesting to see whether these have an effect on stabilizing the quaternary structure of full-length scNOS.

The fate of NO in bacteria and the steady state concentrations of NO within the cell are also areas of interest, as many of the bacteria that express NOS, such as Staphylococcus aureus, Bacillus subtilis and Bacillus anthracis, also express flavohemoglobins, which are highly effective at converting $\mathrm{NO}$ to nitrate through reaction with molecular oxygen and electrons provided by $\mathrm{NAD}(\mathrm{P}) \mathrm{H}[42$, 43]. Thus, these single-celled organisms have the means to both produce and consume NO. How NO production in such situations is balanced by NO consumption through endogenous flavohemoglobins remains to be explored.

\section{ROLES OF BACTERIAL NITRIC OXIDE SYNTHASES}

Exogenous $\mathrm{NO}$ added at modest concentrations to certain Gram-positive bacterial cells can provide prompt but transient protection of cells against oxidative stress [44]. In $B$. subtilis, endogenously-produced $\mathrm{NO}$ acts in two ways to prevent the Fenton reaction, in which $\mathrm{Fe}(\mathrm{II})$ and $\mathrm{Cu}(\mathrm{I})$ reduce hydrogen peroxide to form hydroxyl radical. Redox cycling of the oxidized metals back to the reduced state is mediated by intracellular cysteine, which in turn is maintained in the reduced state by thioredoxin/thioredoxin reductase-catalyzed reduction of cystine to cysteine; the result is further cycles of hydroxyl radical production [44]. NO depletes the cells of cysteine by inhibiting the thiodoxin/thiodoxin reductase pathway, and it directly activates catalase A, which consumes hydrogen peroxide and is inhibited by cysteine.

Evidence that NO generated within the cells also provides protection from oxidative damage comes from experiments that compare the response of NOS deletion and wild type strains of Gram-positive bacteria to a variety of stresses incurred by antibiotics, including oxidative stress [45]. For example, superoxide dismutase A expression in the late $\log$ growth phase of $B$. subtilis is greatly enhanced by endogenous NOS activity, as reporter gene constructs in which the SodA promoter is fused to a reporter betagalactosidase gene show higher levels of expression in wild type B. subtilis but not in the NOS deletion strain. In this case NO acts as a transcriptional regulator, although how it controls gene expression is not yet known.

NOS-derived NO also serves a protective role in Deinocococcus radiodurans, but against UV rather than oxidative stress [46]. This bacteria is especially resistant to harsh environmental conditions and can survive high levels of gamma and UV radiation. UV radiation induces a fourfold increase in NOS transcript levels and an increase in NO production within one hour of exposure. This does not directly protect cells from the damage of UV but instead is a factor in the resumption of the cells to growth afterwards. A proximate signal for resumption of growth is an upregulation of $\operatorname{obg} E$, a small GTP-binding protein found in many bacteria and eukaryotes that may be involved in ribosome assembly as well as other roles that have not yet been fully defined. Interestingly, overexpression of obgE protein in a $D$. radiodurans NOS deletion strain, although protective against UV-irradiation, was incomplete. This could be due to NO acting through additional means other than the obgE pathway.

Apart from generating free $\mathrm{NO}$ to be used in roles such as those described above, the bacterial NOSs may also use NO closer to their site of origin, and perhaps within the active site itself to modify molecules bound close to the heme. The pterin binding site of bacterial NOS is more solvent-exposed than in the mammalian enzymes and can bind a broader range of molecules. Although NO production requires a pterin cofactor bound at these sites, the same may not be true for the production of other reactive nitrogen species such as nitroxide $\left(\mathrm{NO}^{-}\right)$or nitrosonium cation $\left(\mathrm{NO}^{+}\right)$. In these reactions, the pterin binding site may become a second substrate binding site (the L-arginine binding site at the heme being the first binding site) which is modified by the reactive nitrogen species generated at the heme. Several examples of these reactions are known, although their biological significance is not clear. D. radiodurans NOS is able to catalyse selective tryptophan nitration at position-4 when provided with a mammalian reductase domain and 
$\mathrm{NADPH}$, or with $\mathrm{H}_{2} \mathrm{O}_{2}$. These reactions are enhanced in the presence of $D$. radiodurans tryptophanyl-tRNA synthetase II and are inhibited by $\mathrm{H}_{4} \mathrm{~B}$ [47]. Roles for 4-nitrotryptophan in D. radiodurans have not been identified. However, a plant toxin, thaxtomin A of the plant pathogen Streptomyces turgidiscabies, incorporates this moiety, and most thaxtomin biosynthesis depends on the presence of NOS in the bacteria [48]. S. turgidiscabies also synthesizes NO in response to host signals, and might use NO to signal for root development in the host, which would provide the pathogen for more avenues of infection.

Bacterial NOSs may also act against some antibiotics by directly modifying them in addition to the general effect of countering oxidative stress as described above. Acriflavin nitrosation and its subsequent inactivation depend on the presence of NOS, as was demonstrated in an expression system in which $E$. coli cells carried an arabinose-inducible expression vector for $B$. anthracis NOS [45]. It was not determined whether the nitrosating species diffused from the enzyme to the acriflavine, or whether acriflavine was bound to NOS at the pterin binding site; the latter is an attractive mechanism as it would be more effective to have acriflavine bound close to the site where the nitrosating species originates.

Interestingly the ability of the pterin binding site to be the site of chemical modification of bound substrates, first observed in bacterial enzymes may also occur in mammalian NOS as well. Recently the structure of mouse iNOS with an $\mathrm{N}$-nitrosylated $\mathrm{H}_{4} \mathrm{~B}$ cofactor bound at the pterin site was obtained from protein treated with S-nitrosocysteine prior to crystallization [49]. This may be a key intermediate in the regulation of iNOS activity by specific S-nitrosation of the cysteine ligands to the $\mathrm{Zn}(\mathrm{II})$ held at the dimer interface, a reversible modification which destabilizes the dimer and thus inhibits activity. Docking experiments with glutathione suggest that this molecule can bind to iNOS with the thiol in a position suitable for a transnitrosation reaction from NOpterin; it is possible that a similar mechanism may operate in the bacterial NOSs by which they protect against Fenton reactions through S-nitrosation of cysteine, with $\mathrm{H}_{4} \mathrm{~F}$ and cysteine as the partners.

\section{POSSIBLE ROLES OF BACTERIAL NITRIC OXIDE SYNTHASES IN TRANSCRIPTIONAL REGULATION}

NO is a potent signalling molecule even in bacteria that lack the means of producing it. Before the discovery of the bacterial NOSs it was known that bacteria could mount stress responses on exposure to exogenous $\mathrm{NO}$ or phagocytosis by NO-producing macrophages through the soxRS regulon of E. coli [50]. SoxR is a transcription factor that bears an $\mathrm{Fe}_{2} \mathrm{~S}_{2}$ cluster that is activated upon oxidation [51], or by nitrosylation [52]; activated soxR increases the expression of soxS, itself a transcriptional activator of expression of several oxidative stress response genes including $\operatorname{sod} A$, which encodes manganese-containing superoxide dismutase.

Exogenous $\mathrm{NO}$ also produces stress responses in $B$. subtilis and $S$. aureus that are distinct from those of $E$. coli [53]. FeS-containing transcription factors that are activated by nitrosylation are also found in bacteria that possess NOS.
This includes the B. subtilis transcription factor NsrR, which represses expression from the ResDE regulon; expression of this regulon is required for the switch from oxygen to nitrate as the terminal electron acceptor in the respiratory chain under anaerobic conditions. Does endogenously-generated $\mathrm{NO}$ act as a transcriptional regulator in some bacteria? With all the necessary components - NOS for generation, NOresponsive transcription factors, and flavohemoglobins for consumption - this seems likely. No such signalling pathway has yet been defined in detail, but there are some promising leads. A NOS deletion strain of B. subtilis was impaired in sodA expression that normally occurs late in the exponential growth phase [45]. While expression of sodA in B. subtilis differs from that in E. coli [54], it is possible that this also involves transcription factors with FeS switches. Defining such pathways will require careful work to assign roles to NO rather than other agents that can also influence the oxidation state (and therefore the activity) of the $\mathrm{FeS}$ clusters.

\section{BACTERIA, ANIMALS.... WHERE ARE THE OTHER NITRIC OXIDE SYNTHASES?}

An evolutionary chasm of three billion years exists among the organisms that are known to possess NOS. On one side are the bacterial enzymes, largely from Grampositive species and consisting solely of an oxygenase domain dimer. On the other side are the abundant examples of full-length enzymes found in the animal kingdom. Fulllength NOSs are common among the metazoans, and in sufficient numbers to describe their evolutionary descent [31]. Yet apart from the metazoans and bacteria, examples of NOS are rare or elusive. NOS has not yet been identified in higher plants despite much effort and frustration [55]. The number of reports of the presence of NOS in a given species based on indirect evidence such as histological staining, immunoblots with mammalian antibodies, or relatively nonspecific enzyme assays are legion, and frequently are misleading. The only certain way to confirm whether an organism has a NOS is by genetic characterization but even here care must be taken. For example, genomic characterization of the diplomonad protist Giardia lamblia has identified a NOS gene (Genebank Accession Number EDO81993.1), but the annotation is based solely on the presence of a coding region that resembles the NOS reductase domain, and there is no evidence for a sequence homologous to the oxygenase domain.

On the basis that an organism possesses NOS only if it has a gene that encodes a protein which is homologous to the oxygenase domain, there is no evidence yet of NOS in higher plants, while among the fungi, only Aspergillus flavus encodes a full-length NOS, although the enzyme has not yet been characterized. The slime mold Physarum polycephalum possesses two full-length NOS isotypes that are induced under nutrient restriction and are involved in sporulation [56]. These have been cloned and the recombinant proteins have been expressed and partially characterized. While they possess a calmodulin binding site their reductase domains lack the autoinhibitory loop found in the NOS1 and NOS3 isotypes of mammals.

An exciting recent development which may help to bridge the metazoans and the bacteria NOSs comes from the 
recently described genome of the amoeboflagellate Naegleria gruberi, a single-celled, free-living organism thought to resemble the ancestor of all extant eukaryotes [57]. Although its genome size is only $41 \mathrm{Mbp}$, it encodes over 15,000 proteins, which is comparable to the number of proteins encoded by the larger genomes of metazoans such as humans ( 25,000 genes) and fruit fly $(13,739$ genes) [58, 59]. Interestingly the draft genome sequence of $N$. gruberi includes a gene which encodes a 474 residue protein that is homologous to the NOS oxygenase domain, with a wellconserved sequence particularly in the residues of the cofactor binding pockets (NCBI Accession Number XP_002679491.1; UniProtKB Accession Number D2V8T5). N. gruberi also has genes that encode cytochrome P450 reductase-like enzymes as well as other flavoproteins that could possibly serve as electron donors to its NOS. The presence of a single-domain NOS in $N$. gruberi supports the idea that the gene fusion which gave rise to the full-length NOS enzymes characteristic of the metazoans arose after the evolution of eukaryotes. Nothing is known of the role of NOS, nor of biological effects NO itself, in this organism. As $N$. gruberi is a unicellular organism and its NOS does not bear a reductase domain, its functional roles may resemble those of bacterial NOS, and it may produce $\mathrm{NO}$ at comparable rates. In support of this hypothesis, the $N$. gruberi NOS sequence, like the bacterial enzymes, has a conserved isoleucine at position 242, corresponding to isoleucine-218 in B. subtilis NOS and valine-346 in mouse iNOS, which, as noted above, influences the rate of $\mathrm{NO}$ dissociation from heme [24]. We await the characterization of the $N$. gruberi enzyme; one protein may not fill the chasm between bacterial and animal NOSs, but it can provide a significant link.

\section{ACKNOWLEDGEMENT}

NSERC Discovery Grant 216849 and CFI Grant 2318.

\section{CONFLICT OF INTEREST}

The authors reports no conflict of interest.

\section{REFERENCES}

[1] Stone, J. R.; Marletta, M. A. Soluble Guanylate cyclase from bovine lung: activation with nitric oxide and carbon monoxide and spectral characterization of the ferrous and ferric states. Biochemistry, 1994, 33, 5636-5640.

[2] Ignarro, L. J.; Degnan, J. N.; Baricos, W. H.; Kadowitz, P. J.; Wolin, M. S. Activation of purified guanylate cyclase by nitric oxide requires heme comparison of heme-deficient, hemereconstituted and heme-containing forms of soluble enzyme from bovine lung. Biochim Biophys Acta (BBA) - Gen Subjects., 1982, 718, 49-59.

[3] Pellat, C.; Henry, Y.; Drapier, J.-C. IFN-[gamma]-activated macrophages: Detection by electron paramagnetic resonance of complexes between L-Arginine-derived nitric oxide and non-heme iron proteins. Biochem Biophys, Res, Commun., 1990, 166, 119125.

[4] Hidalgo, E.; Demple, B. An iron-sulfur center essential for transcriptional activation by the redox-sensing SoxR protein. EMBO J., 1994, 13, 138-146.

[5] Trageser, M.; Unden, G. Role of cysteine residues and of metal ions in the regulatory functioning of FNR. the transcriptional regulator of anaerobic respiration in Escherichia coli, Mol Microbiol., 1989, 3, 593-599.

[6] Lepoivre, M.; Fieschi, F.; Coves, J.; Thelander, L.; Fontecave, M. Inactivation of ribonucleotide reductase by nitric oxide. Biochem. Biophys, Res, Commun., 1991, 179, 442-448.
Stamler, J. S.; Simon, D. I.; Osborne, J. A.; Mullins, M. E.; Jaraki, O.; Michel, T.; Singel, D. J.; Loscalzo, J. S-nitrosylation of proteins with nitric oxide: synthesis and characterization of biologically active compounds. Proc. Natl. Acad. Sci. USA, 1992, 89, 444-448.

[8] Beckman, J. S.; Beckman, T. W.; Chen, J.; Marshall, P. A.; Freeman, B. A. Apparent hydroxyl radical production by peroxynitrite: implications for endothelial injury from nitric oxide and superoxide. Proc. Natl. Acad. Sci. USA, 1990, 87, 1620-1624.

[9] Liu, J.; Garcia-Cardena, G.; Sessa, W. C. Palmitoylation of endothelial nitric oxide synthase is necessary for optimal stimulated release of nitric oxide: implications for caveolae localization. Biochemistry., 1996, 35, 13277-13281.

[10] Brenman, J. E.; Chao, D. S.; Gee, S. H.; McGee, A. W.; Craven, S E.; Santillano, D. R.; Wu, Z.; Huang, F.; Xia, H.; Peters, M. F.; Froehner, S. C.; Bredt, D. S. Interaction of nitric oxide synthase with the postsynaptic density protein psd-95 and [alpha]1syntrophin mediated by pdz domains. Cell, 1996, 84, 757-767.

[11] Chanrion, B.; Mannoury la Cour, C.; Bertaso, F.; Lerner-Natoli, M.; Freissmuth, M.; Millan, M. J.; Bockaert, J.; Marin, P. Physical interaction between the serotonin transporter and neuronal nitric oxide synthase underlies reciprocal modulation of their activity. Proc. Natl. Acad. Sci. USA, 2007, 104, 8119-8124.

[12] Raman, C. S.; Li, H.; Martasek, P.; Kral, V.; Masters, B. S.; Poulos, T. L. Crystal structure of constitutive endothelial nitric oxide synthase: a paradigm for pterin function involving a novel metal center. Cell, 1998, 95, 939-950.

[13] Crane, B. R.; Arvai, A. S.; Ghosh, D. K.; Wu, C.; Getzoff, E. D.; Stuehr, D. J.; Tainer, J. A. Structure of nitric oxide synthase oxygenase dimer with pterin and substrate. Science, 1998, 279, 2121-2126.

[14] Pant, K.; Bilwes, A. M.; Adak, S.; Stuehr, D. J.; Crane, B. R. Structure of a nitric oxide synthase heme protein from Bacillus subtilis. Biochemistry, 2002, 41, 11071-11079.

[15] Bird, L. E.; Ren, J.; Zhang, J.; Foxwell, N.; Hawkins, A. R.; Charles, I. G.; Stammers, D. K. Crystal structure of sanos a bacterial nitric oxide synthase oxygenase protein from staphylococcus aureus. Structure, 2002, 10, 1687-1696.

[16] Pufahl, R. A.; Nanjappan, P. G.; Woodard, R. W.; Marletta, M. A Mechanistic probes of N-hydroxylation of L-arginine by the inducible nitric oxide synthase from murine macrophages. Biochemistry, 1992, 31, 6822-6828.

[17] Daff, S. NO synthase: structures and mechanisms. Nitric Oxide, 2010, 23, 1-11.

[18] Crane, B. R.; Sudhamsu, J.; Patel, B. A. Bacterial nitric oxide synthases. Annu. Rev. Biochem., 2010, 79, 445-470.

[19] Wei, C.-C.; Wang, Z.-Q.; Hemann, C.; Hille, R.; Stuehr, D. J. A tetrahydrobiopterin radical forms and then becomes reduced during $\mathrm{n}\{$ omega $\}$-hydroxyarginine oxidation by nitric-oxide synthase. $J$. Biol. Chem., 2003, 278, 46668-46673.

[20] Wei, C.-C.; Wang, Z.-Q.; Wang, Q.; Meade, A. L.; Hemann, C.; Hille, R.; Stuehr, D. J. Rapid kinetic studies link tetrahydrobiopterin radical formation to heme-dioxy reduction and arginine hydroxylation in inducible nitric-oxide synthase. J. Biol. Chem., 2001, 276, 315-319.

[21] Sudhamsu, J.; Crane, B. R. Structure and reactivity of a thermostable prokaryotic nitric-oxide synthase that forms a longlived oxy-heme complex. J. Biol. Chem., 2006, 281, 9623-9632.

[22] Adak, S.; Aulak, K. S.; Stuehr, D. J. Direct Evidence for nitric oxide production by a nitric-oxide synthase-like protein from bacillus subtilis. J. Biol. Chem., 2002, 277, 16167-16171.

[23] Gautier, C.; Mikula, I.; Nioche, P.; Martasek, P.; Raman, C. S. Slama-Schwok, A. Dynamics of NO rebinding to the heme domain of NO synthase-like proteins from bacterial pathogens. Nitric Oxide., 2006, 15, 312-327.

[24] Wang, Z.-Q.; Wei, C.-C.; Sharma, M.; Pant, K.; Crane, B. R.; Stuehr, D. J. A conserved val to ile switch near the heme pocket of animal and bacterial nitric-oxide synthases helps determine their distinct catalytic profiles. J. Biol. Chem., 2004, 279, 19018-19025.

[25] Lang, J.; Driscoll, D.; GÈlinas, S.; Rafferty, S. P.; Couture, M. Trp180 of endothelial NOS and Trp56 of bacterial saNOS modulate sigma bonding of the axial cysteine to the heme. $J$. Inorg. Biochem., 2009, 103, 1102-1112.

[26] Chartier, F. J.; Couture, M. Interactions between substrates and the haem-bound nitric oxide of ferric and ferrous bacterial nitric oxide synthases. Biochem, J 2007, 401, 235-245. 
[27] Chartier, F. J.; Couture, M. Stability of the heme environment of the nitric oxide synthase from Staphylococcus aureus in the absence of pterin cofactor. Biophys. J., 2004, 87, 1939-1950.

[28] Sheta, E. A.; McMillan, K.; Masters, B. S. Evidence for a bidomain structure of constitutive cerebellar nitric oxide synthase. J. Biol. Chem., 1994, 269, 15147-15153.

[29] Shaul, P. W.; Smart, E. J.; Robinson, L. J.; German, Z.; Yuhanna, I. S.; Ying, Y.; Anderson, R. G. W.; Michel, T. Acylation targets endothelial nitric-oxide synthase to plasmalemmal caveolae. $J$. Biol. Chem., 1996, 271, 6518-6522.

[30] Garcia-Cardena, G.; Fan, R.; Stern, D. F.; Liu, J.; Sessa, W. C. Endothelial nitric oxide synthase is regulated by tyrosine phosphorylation and interacts with caveolin-1. J. Biol. Chem., 1996, 271, 27237-27240.

[31] Andreakis, N.; D'Aniello, S.; Albalat, R.; Patti, F. P.; GarciaFernandez, J.; Procaccini, G.; Sordino, P.; Palumbo, A. Evolution of the nitric oxide synthase family in metazoans. Mol. Biol. Evol., 2011, 28(1), 163-179.

[32] Gusarov, I.; Starodubtseva, M.; Wang, Z.-Q.; McQuade, L.; Lippard, S. J.; Stuehr, D. J.; Nudler, E. Bacterial nitric-oxide synthases operate without a dedicated redox partner. J. Biol. Chem., 2008, 283, 13140-13147.

[33] Wang, Z.-Q.; Lawson, R. J.; Buddha, M. R.; Wei, C.-C.; Crane, B. R.; Munro, A. W.; Stuehr, D. J. Bacterial flavodoxins support nitric oxide production by bacillus subtilis nitric-oxide synthase. J. Biol. Chem., 2007, 282, 2196-2202.

[34] Reece, S. Y.; Woodward, J. J.; Marletta, M. A. Synthesis of nitric oxide by the nos-like protein from deinococcus radiodurans: a direct role for tetrahydrofolate. Biochemistry, 2009, 48, 5483-5491.

[35] Stasiv, Y.; Regulski, M.; Kuzin, B.; Tully, T.; Enikolopov, G. The drosophila nitric-oxide synthase gene (dnos) encodes a family of proteins that can modulate nos activity by acting as dominant negative regulators. J.Biolo. Chem., 2001, 276, 42241-42251.

[36] Stasiv, Y.; Kuzin, B.; Regulski, M.; Tully, T.; Enikolopov, G. Regulation of multimers via truncated isoforms: a novel mechanism to control nitric-oxide signaling. Genes Dev., 2004, 18, 1812-1823.

[37] Agapie, T.; Suseno, S.; Woodward, J. J.; Stoll, S.; Britt, R. D.; Marletta, M. A. NO formation by a catalytically self-sufficient bacterial nitric oxide synthase from sorangium cellulosum. Proc. Natl. Acad. Sci. USA, 2009, 106, 16221-16226.

[38] Deutscher, J.; Saier, J. M. H. Ser/Thr/Tyr protein phosphorylation in bacteria ,äì for long time neglected. Now Well Established. $J$. Mol. Microbiol. Biotech., 2005, 9, 125-131.

[39] Cozzone, A. J. Role of protein phosphorylation on serine/threonine and tyrosine in the virulence of bacterial pathogens. J. Mol. Microbiol. Biotech.,, 2005, 9, 198-213.

[40] Pant, K.; Crane, B. R. Structure of a loose dimer: an intermediate in nitric oxide synthase assembly. J. Mol. Biol., 2005, 352, 932-940.

[41] Combet, C.; Blanchet, C.; Geourjon, C.; Deleage, G. NPS@: network protein sequence analysis. TIBS 2000, 25, 147-150.

[42] Nobre, L. S.; GonÁalves, V. L.; Saraiva, L. M.; Robert, K. P. Flavohemoglobin of staphylococcus aureus. In. Method. Enzymolo., Academic Press, 2008, pp. 203-216.

[43] Ilari, A.; Boffi, A.; Robert, K. P. Structural studies on flavohemoglobins. In. Method. Enzymolo., Academic Press. 2008, pp. 187-202.

[44] Gusarov, I.; Nudler, E. NO-mediated cytoprotection: instant adaptation to oxidative stress in bacteria. Proc. Natl. Acad. Sci., U $S$ A 2005, 102, 13855-13860.
[45] Gusarov, I.; Shatalin, K.; Starodubtseva, M.; Nudler, E. Endogenous nitric oxide protects bacteria against a wide spectrum of antibiotics. Science, 2009, 325, 1380-1384.

[46] Patel, B. A.; Moreau, M.; Widom, J.; Chen, H.; Yin, L.; Hua, Y.; Crane, B. R. Endogenous nitric oxide regulates the recovery of the radiation-resistant bacterium Deinococcus radiodurans from exposure to UV light. Proc. Natl. Acad. Sci., US A, 2009, 106, 18183-18188.

[47] Buddha, M. R.; Tao, T.; Parry, R. J.; Crane, B. R. Regioselective nitration of tryptophan by a complex between bacterial nitric-oxide synthase and tryptophanyl-tRNA synthetase. J. Biol. Chem., 2004, 279, 49567-49570.

[48] Kers, J. A.; Wach, M. J.; Krasnoff, S. B.; Widom, J.; Cameron, K. D.; Bukhalid, R. A.; Gibson, D. M.; Crane, B. R.; Loria, R. Nitration of a peptide phytotoxin by bacterial nitric oxide synthase. Nature, 2004, 429, 79-82.

[49] Rosenfeld, R. J.; Bonaventura, J.; Szymczyna, B. R.; MacCoss, M. J.; Arvai, A. S.; Yates, J. R., 3rd; Tainer, J. A.; Getzoff, E. D Nitric-oxide synthase forms N-NO-pterin and S-NO-cys: implications for activity, allostery, and regulation. J. Biol. Chem., 2010, 285, 31581-31589.

[50] Nunoshiba, T.; deRojas-Walker, T.; Wishnok, J. S.; Tannenbaum, S. R.; Demple, B. Activation by nitric oxide of an oxidative-stress response that defends Escherichia coli against activated macrophages. Proc. Natl. Acad. Sci. USA., 1993, 90, 9993-9997.

[51] Gaudu, P.; Weiss, B. SoxR, a [2Fe-2S] transcription factor, is active only in its oxidized form. Proc. Natl. Acad. Sci. USA., 1996, 93, 10094-10098.

[52] Ding, H.; Demple, B. Direct nitric oxide signal transduction via nitrosylation of iron-sulfur centers in the SoxR transcription activator. Proc. Natl. Acad. Sci. USA., 2000, 97, 5146-5150.

[53] Hochgrafe, F.; Wolf, C.; Fuchs, S.; Liebeke, M.; Lalk, M. Engelmann, S.; Hecker, M. Nitric oxide stress induces different responses but mediates comparable protein thiol protection in bacillus subtilis and staphylococcus aureus. J. Bacteriol., 2008, 190, 4997-5008.

[54] Inaoka, T.; Matsumura, Y.; Tsuchido, T. Molecular cloning and nucleotide sequence of the superoxide dismutase gene and characterization of its product from Bacillus subtilis. J. Bacteriol. 1998, 180, 3697-3703.

[55] Zemojtel, T.; Frohlich, A.; Palmieri, M. C.; Kolanczyk, M. Mikula, I.; Wyrwicz, L. S.; Wanker, E. E.; Mundlos, S.; Vingron, M.; Martasek, P.; Durner, J. Plant nitric oxide synthase: a neverending story? Trends Plant Sci., 2006, 11, 524-525; author reply 526-528.

[56] Messner, S.; Leitner, S.; Bommassar, C.; Golderer, G.; Grobner, P.; Werner, E. R.; Werner-Felmayer, G. Physarum nitric oxide synthases: genomic structures and enzymology of recombinant proteins. Biochem. J., 2009, 418, 691-700.

[57] Fritz-Laylin, L. K.; Prochnik, S. E.; Ginger, M. L.; Dacks, J. B.; Carpenter, M. L.; Field, M. C.; Kuo, A.; Paredez, A.; Chapman, J.; Pham, J.; Shu, S.; Neupane, R.; Cipriano, M.; Mancuso, J.; Tu, H.; Salamov, A.; Lindquist, E.; Shapiro, H.; Lucas, S.; Grigoriev, I. V.; Cande, W. Z.; Fulton, C.; Rokhsar, D. S.; Dawson, S. C. The genome of naegleria gruberi illuminates early eukaryotic versatility. Cell, 2010, 140, 631-642.

[58] Adams, M. D., et al. The genome sequence of drosophila melanogaster. Science 2000, 287, 2185-2195.

[59] Finishing the euchromatic sequence of the human genome. Nature., 2004, 431, 931-945. 